\title{
Prevalence of microalbuminuria in type 2 diabetes patients in Tirana, a preliminary multicenter study
}

\author{
Nevi Pasko ${ }^{1^{*}}$, Florian Toti $^{2}$, Ervin Zekollari ${ }^{1}$, Arjana Strakosha ${ }^{1}$, Viola Kacori ${ }^{1}$, \\ Nestor Thereska ${ }^{1}$ \\ ${ }^{1}$ Department of Nephrology Dialysis and Transplant, University Hospital Center, Tirana, Albania; \\ *Corresponding Author: nevipasko@hotmail.com \\ ${ }^{2}$ Department of Endocrinology, University Hospital Center, Tirana, Albania
}

Received 29 May 2013; revised 30 June 2013; accepted 7 July 2013

Copyright (C) 2013 Nevi Pasko et al. This is an open access article distributed under the Creative Commons Attribution License, which permits unrestricted use, distribution, and reproduction in any medium, provided the original work is properly cited.

\section{ABSTRACT}

Background: Microalbuminuria is often the first sign of renal involvement predicting overt nephropathy. For this reason, monitoring microalbuminuria and other risk factors associated with this condition is important to take measures to prevent or postpone overt nephropathy. This study aimed to investigate the prevalence of microalbuminuria in type 2 diabetes patients attending three diabetes centers in Tirana city. Patients and Methods: Two hundred and twenty patients with type 2 diabetes attending diabetes centers in Tirana were recruited in this crosssectional study. Medical records were used to collect data on duration of diabetes, waist circumference, history of hypertension, smoking. Blood samples were drawn after $12 \mathrm{~h}$ overnight fasting to measure glycosylated hemoglobin (HbA1c), serum cholesterol, triglyceride and creatinine. Microalbuminuria was assessed using dipstick kits in early morning urine samples. Results: The prevalence of normoalbuminuria

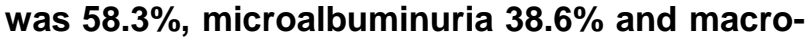
albuminuria $3.1 \%$. Systolic and diastolic blood pressure $(p<0.01)$, HbA1c $(p<0.01)$ and fasting plasma glucose $(p<0.001)$ were significantly higher in microalbuminuric than in normoalbuminuric subjects. Multiple logistic regression analysis using microalbuminuria as the dependent variable in males shows that independent risk factors for diabetes patients with microalbuminuria were duration of diabetes, systolic blood pressure and waist circumference. We found that the OR for microalbuminuria became statistically significantly increased only at 16 years after the diagnosis of type 2 diabetes.
At this time, $43.7 \%$ of patients had microalbuminuria. Conclusions: We found a high proportion of type 2 diabetes patients with microalbuminuria which raises implications for health policy in Albania. This calls for early detection and good control of diabetes to reduce the burden of diabetic kidney disease in the future. Screening programs and optimized control of modifiable risk factors are needed to reduce the risk of diabetic nephropathy.

Keywords: Microalbuminuria; Type 2 Diabetes; Epidemiology; Albania

\section{INTRODUCTION}

Recent figures released by the International Diabetes Federation confirm the enormity of the diabetes epidemic and indicate that the number of patients with renal failure due to diabetes will continue to increase dramatically. Diabetic nephropathy it is also one of the most significant long-term complications in terms of morbidity and mortality for individual patients with diabetes. Several small and large scale trials in the United States, Canada and Europe have tried to evaluate the incidence and prevalence of diabetic nephropathy [1]. In United States diabetes is responsible for $30 \%-40 \%$ of all end-stage renal disease (ESRD) cases. As in other developing countries, the prevalence of diabetes in Albania has increased rapidly [2] and as a consequence the number of patients with diabetic kidney disease is expected to greatly increase with important implications for health policy. According to the International Diabetes Federation [3], in 2012 there were about 65,000 cases of diabetes in Albania (approximately $2.3 \%$ of the population). The Atlas forecasts that the prevalence rate will rise to 


\section{$3.6 \%$ by 2030 .}

Over the last decade, Albania has undergone profound political and economic changes. A greater government support over the last years has resulted in a continuous increase in the number of patients receiving RRT. In Albania, approximately $14 \%$ of patients on hemodialysis are diabetics. Kidney diseases are often detected too late, when the patient is already in ESRD and need dialysis treatment. The natural history of diabetic nephropathy is well understood. The studies have demonstrated that without specific intervention, the glomerular filtration rate (GFR) will continue to decrease over several years at a rate that is highly variable in patients with either type 1 or type 2 diabetes [4]. Microalbuminuria is often the first sign of renal involvement predicting overt nephropathy [5]. For this reason, measurement of urine albumin is often used as a sensitive marker and predictor of overt nephropathy in patients with diabetes [6]. Monitoring microalbuminuria and other risk factors associated with this condition is important to take measures to prevent or postpone overt nephropathy [7]. Measurement of microalbuminuria in a $24 \mathrm{~h}$ urine collection is the gold standard [8], though an alternative is a spot urine sample adjusted to determine creatininuria. A spot urine collection is considered positive if the albumin/creatinine ratio (ACR) is $30-299 \mathrm{mg}$ albumin/g creatinine $[9,10]$. In many studies, ACR has been recognized as a valid method for screening populations $[9,11]$.

The primary aim of this study was to evaluate the prevalence of microalbuminuria and renal impairment in patients with type 2 diabetes without known proteinuria. The results could be important in increasing primary physicians' awareness of the importance of regular urinary albumin screening in order to improve medical care of diabetic patients.

\section{PATIENTS AND METHODS}

During the period March-November 2011 we collected data of 220 patients with type 2 diabetes attending three different polyclinics in Tirana districts of Albania. Ethical approval was obtained from the National Medical Ethics Committee. We recruited patients without known proteinuria or other kidney disease, aged between 40 and 70 years. We excluded pregnant women and patients with acute infections.

We used their medical records to obtain information regarding their gender, age, height and weight to calculate body mass index, waist circumference, duration of diabetes, history of hypertension and drugs used, and history of smoking. Patients were defined as hypertensive if they were undergoing treatment with antihypertensive drugs or if their untreated systolic blood pressure was $>130 \mathrm{mmHg}$ and/or diastolic blood pressure $>85$ $\mathrm{mmHg}$. Normal waist circumference was defined as
$<102 \mathrm{~cm}$ for males or $<88 \mathrm{~cm}$ for females. A sample of blood was drawn after overnight fasting for $12 \mathrm{~h}$ to measure glycosylated hemoglobin (HbAlc), serum cholesterol, triglyceride and creatinine levels. Microalbuminuria was assessed using Bayer DCA 2000 Microalbumin test kits in urine samples collected in the early morning. Patients were classified as normoalbuminuric if their ACR was $<30 \mathrm{mg}$ albumin $/ \mathrm{g}$ creatinine or microalbuminuric if their ACR was $30-300 \mathrm{mg}$ albumin $/ \mathrm{g}$ creatinine. When the ACR was $>300 \mathrm{mg}$ albumin $/ \mathrm{g}$ creatinine on two consecutive tests, patients were considered to have macroalbuminuria or overt nephropathy.

Data analysis was conducted using the Statistical Package for Social Sciences (SPSS) for Windows version 11 . The chi-square test and logistic regression analysis were used to determine correlations between microalbuminuria and other risk factors.

\section{RESULTS}

Two hundred and twenty type 2 diabetes patients (116 females and 104 males) were included in this study. Their mean age was $60.3 \pm 9.2$ years. Of the 220 subjects, $38(17.2 \%)$ were found to have diabetes at their first visit to their health care provider and fourteen of these had microalbuminuria at the time of diagnosis. The mean duration of diabetes was $7.2 \pm 3.9$ years. The prevalence of normoalbuminuria, microalbuminuria and macroalbuminuria were $58.3 \%, 38.6 \%$ and $3.1 \%$, respectively.

Table 1 shows clinical characteristics of patients stratified by microalbuminuria status. Macroalbuminuric patients $(3.1 \%$ of the study population) had creatinine serum levels $>1.5 \mathrm{mg} / \mathrm{dl}$. The prevalence of microalbuminuria was $40.5 \%$ in males and $41.1 \%$ in females. There was no significant difference between males and females in the prevalence of macroproteinuria or microproteinuria.

The prevalence of microalbuminuria increased with the duration of diabetes (Figure 1). Taking $\leq 5$ years as the reference value, odds ratios (ORs) for durations of 6 $10,11-15,16-20$ and $>20$ years were calculated. The OR for microalbuminuria became statistically significantly increased 16 years after the diagnosis of type 2 diabetes, by which time $48.2 \%$ of patients had microalbuminuria $(p<0.001)$.

Among both male and female, those with ACR $\geq 30$ $\mathrm{mg}$ albumin/g creatinine had significantly increased systolic and diastolic blood pressure compared with normoalbuminuric subjects $(p<0.01)$. HbA1c concentrations and fasting plasma glucose levels were significantly higher with microalbuminuria than with normoalbuminuria $(p<0.001)$. Taking an HbA1c concentration of $<7 \%$ as the reference value, there was a significant increase in the OR for microalbuminuria (OR: 2.0, 95\% confidence interval (CI): 1.04 - 3.87) in microalbuminuric patients 
Table 1. Clinical characteristics of the study subjects.

\begin{tabular}{cccc}
\hline & Normoalbuminuria & Microalbuminuria & Macroalbuminuria \\
\hline Number & $128(58.3 \%)$ & $85(38.6 \%)$ & $7(3.1 \%)$ \\
Gender (M/F) & $50 / 78$ & $39 / 46$ & $2 / 5$ \\
Age (years) & $58.3 \pm 7.6$ & $57.5 \pm 8.7$ & $59.5 \pm 4.8$ \\
HbA1c & $7.2 \pm 1.8$ & $8.2 \pm 1.3$ & $8.4 \pm 1.2$ \\
Waist circumference (male) & $98 \pm 7.2$ & $101 \pm 8.1$ & $115 \pm 6.3$ \\
Waist circumference (female) & $81 \pm 5.6$ & $91 \pm 2.3$ & $95 \pm 4.6$ \\
Cholesterol & $185 \pm 22.9$ & $187 \pm 25.6$ & $195 \pm 33.9$ \\
Triglyceride & $135 \pm 54.6$ & $154 \pm 78.3$ & $186 \pm 68.9$ \\
Smoking & $26(20.1 \%)$ & $19(22.3 \%)$ & $4(57.1 \%)$ \\
Duration of diabetes & $7.49 \pm 5.6$ & $8.2 \pm 4.5$ & $9.6 \pm 7.8$ \\
Systolic blood pressure & $125 \pm 4.8$ & $139 \pm 7.9$ & $145 \pm 15.6$ \\
Diastolic blood pressure & $70 \pm 9.5$ & $85 \pm 8.5$ & $90 \pm 9.7$ \\
RAS blockades use & $41(32.1 \%)$ & $57(67 \%)$ & $6(85.7 \%)$ \\
\hline
\end{tabular}

Abbreviations: HbA1c, glycosylated hemoglobin; M/F, male/female; RAS, renin angiotensin system.

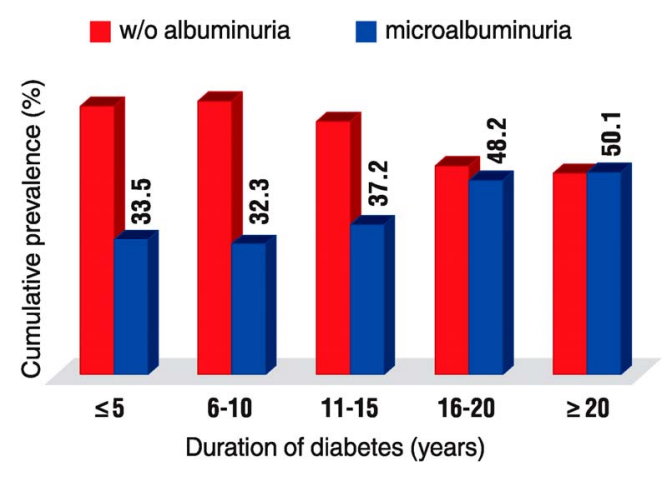

Figure 1. Cumulative prevalence of microalbuminuria in relation to duration of diabetes. The prevalence of microalbuminuria increased with duration of diabetes and the association was statistically significant 16 years after diagnosis; $48.2 \%$ of patients had microalbuminuria at this time, though the increase remained unchanged thereafter.

with $\mathrm{HbA} 1 \mathrm{c}$ levels of $8 \%-10 \%$.

Multiple logistic regression analysis using microalbuminuria as the dependent variable in males shows that independent risk factors for male diabetes patients with microalbuminuria were duration of diabetes, systolic blood pressure (OR: $2.88,95 \%$ CI: $1.85-6.85$ ) and waist circumference (OR: $2.15,95 \%$ CI: $1.01-5.45$ ). While in females, there were significant associations with poor glycemic control (OR: 4.51, 95\% CI: 1.45 - 13.98), duration of diabetes and waist circumference (OR: 4.87, 95\% CI: 1.80 - 13.11). The following factors were used as independent variables: duration of diabetes in 5 year intervals; systolic blood pressure $>140 \mathrm{~mm} \mathrm{Hg}$ and/or diastolic blood pressure $>90 \mathrm{~mm} \mathrm{Hg}$; waist circumference
$>102 \mathrm{~cm}$ in males or $>88 \mathrm{~cm}$ in females; and HbAlc.

\section{DISCUSSION}

Over the past decades, there has been a significant worldwide increase in the incidence of diabetes mellitus [3]. This current global epidemic is associated with an increase of cardiovascular diseases that primarily accounts for the increase in morbidity and mortality seen in patients with diabetes $[12,13]$. The high prevalence of microvascular complications of diabetes such as diabetic nephropathy means that the number of patients with end-stage renal disease (ESRD) due to diabetes will also increase dramatically [14]. Hence, diabetes, and especially type 2 diabetes, is becoming the main reason for patients to start renal replacement therapy $[15,16]$.

In general, microalbuminuria is a sensitive marker for damage induced by diabetes [17]. Epidemiologic studies have shown that identifying and monitoring patients with microalbuminuria is important because its treatment can prevent or postpone overt nephropathy $[17,18]$.

In the present cross-sectional study, we investigated the prevalence of microalbuminuria and its association with potential risk factors in 220 type 2 diabetes patients in Tirana district. Standard assays for urinary microalbuminuria are not widely available in Albanian laboratories, which made the study of diabetic nephropathy difficult in this country. We measured microalbuminuria using Bayer dipsticks, which is an easy and accurate method.

Of our study population, $40.8 \%$ had microalbuminuria, which is similar to the prevalence in other European countries [19]. Data from the DEMAND study, a large 
cohort of type 2 diabetes patients, found the prevalence of normoalbuminuria and microalbuminuria to be $51 \%$ and $39 \%$, respectively [20]. An earlier study conducted on the framework of the ALBDIAB study of 2004 [21] showed that $9.4 \%$ of diabetes patients had diabetic nephropathy. However, that study did not include microalbuminuria in the prevalence of nephropathy and dealt only with overt nephropathy, which could be the reason for the low reported prevalence of kidney damage. In the present study, we did not find any difference between males and females regarding the presence of microalbuminuria, which is similar to the findings reported by Mather et al. for European diabetes patients [22].

The microalbuminuric patients in our study had a longer duration of diabetes than the normoalbuminuric group, consistent with findings from other studies [23]. We found that the OR for microalbuminuria became statistically significantly increased only at 16 years after the diagnosis of type 2 diabetes. At this time, $43.7 \%$ of patients had microalbuminuria; this figure remained constant thereafter. However, it is well known that the duration of disease is difficult to establish in type 2 diabetes. Microalbuminuric patients in the present study had higher systolic and diastolic blood pressure, suggesting that systolic blood pressure is a significant risk factor for diabetic nephropathy, but multivariate analysis did not confirm this finding. The reason may be the definition used for hypertension, because the patients we classified as non-hypertensive were not reevaluated. Microalbuminuric patients tend to have higher levels of cholesterol and triglyceride, but no independent significant association was observed in the present study. A possible explanation for this result may be the use of statins in this group. In most studies, a good correlation has been observed between the prevalence of microalbuminuria and cholesterol and triglyceride levels. In a prospective observational study, Gall et al. found that baseline cholesterol was an independent risk factor for the development of microalbuminuria [24].

Several limitations were encountered in this study. First, the study was not population-based and only patients who presented at diabetes centers were included. This may have introduced referral bias and it would therefore be difficult to extend our findings to the general population of diabetes patients. Second, we had difficulties determining the duration of diabetes. Third, the cross-sectional nature of the study design limits the reliability of the observed associations between risk factors and diabetic nephropathy. Despite these limitations, the high number of patients with microalbuminuria raises implications for future health policies. Because microalbuminuria is widely used as a sensitive risk marker to identify those at risk for renal dysfunction [25], screening programs should be implemented at an early stage to prevent or postpone ESRD. However, a recent study by Thomas et al. showed that renal impairment in type 2 diabetes is not always preceded by albuminuria and other markers are therefore needed to monitor renal function [26]. Prospective studies should be undertaken to confirm the relationship between risk factors and the progression of diabetic nephropathy, and to monitor renal dysfunction in these patients.

\section{CONCLUSIONS}

In conclusion, the prevalence of microalbuminuria in this Tirana city based study among type 2 diabetic patients is $40.8 \%$ and the risk factors are similar to that reported among Europeans. In males, high systolic blood pressure, duration of diabetes and central obesity were associated with development of microalbuminuria; in females, poor glycemic control, duration of diabetes and waist circumference were associated factors. Given the increasing prevalence of diabetes as a cause of end stage renal disease, this could place considerable burden on the health budgets of our country. This calls for early detection and good control of diabetes to reduce the burden of diabetic kidney disease in the future.

Obviously, diabetic patients with microalbuminuria are at greater risk to develop nephropathy or other cardiovascular diseases. According to the American Diabetes Association, type 2 diabetes patients should be screened annually for microalbuminuria. Unfortunately, we are far from routine screening and other recommended goals for these patients in Albania. In the present study, we investigated the prevalence of microalbuminuria in Albanian diabetes patients and emphasize the need for routine screening. This can be done with simple and inexpensive urine tests and effective therapy should start with early diagnosis of kidney damage.

\section{REFERENCES}

[1] de Boer, I.H., Rue, T.C., Hall, Y.N., Heagerty, P.J., Weiss, N.S. and Himmelfarb, J. (2011) Temporal trends in the prevalence of diabetic kidney disease in the United States. Journal of the American Medical Association, 305, 2532 2539. doi:10.1001/jama.2011.861

[2] Shapo, L., McKee, M., Coker, R. and Ylli, A. (2004) Type 2 diabetes in Tirana city, Albania: A rapid increase in a country in transition. Diabetic Medicine, 21, 77-83. doi:10.1046/j.1464-5491.2003.01093.x

[3] International Diabetes Federation (2012) IDF diabetes atlas [Homepage on the Internet]. http://www.idf.org/diabetesatlas

[4] Mongensen, C.E., Keane, W.F., Beneth, P.H., Parving, H.H. and Passa, P. (1995) Prevention of diabetic renal disease with special reference to microalbuminuria and macroalbuminuria. Lancet, 346, 1080-1084. doi:10.1016/S0140-6736(95)91747-0 
[5] Jong, P.E., Hillege, H.L., Joan, P.-S. and Zeeuw, D. (2003) Screening for microalbuminuria in the general population: A tool to detect subjects at increased risk for progressive renal failure in an early phase? Nephrology Dialysis Transplantation, 18, 10-13. doi:10.1093/ndt/18.1.10

[6] Hiddo, J., Heerspink, L., Holtkamp, F. and Ravid, M. (2011) Monitoring kidney function and albuminuria in patients with diabetes. Diabetes Care, 34, 325-329. doi: $10.2337 / \mathrm{dc} 11-\mathrm{s} 247$

[7] de Zeeuw, D., Remuzzi, G. and Parving, H.H. (2004) Proteinuria, a target for renoprotection in patients with type 2 diabetic nephropathy: Lessons from RENAAL. Kidney International, 65, 2309-2320. doi:10.1111/j.1523-1755.2004.00653.x

[8] Rowe, D.J., Dawnay, A. and Watts, G.F. (1990) Microalbuminuria in diabetic subjects. Annals of Clinical Biochemistry, 27, 297-312.

[9] Croal, B.L., Mutch, W.J., Clark, B.M., Dickie, A., Church, J. and Noble, D. (2001) The clinical application of a urine albumin: Creatinine ratio point-of-care device. Clinica Chimica Acta, 307, 15-21. doi:10.1016/S0009-8981(01)00450-8

[10] Marshall, S.M. (1991) Screening for MAU: Which measurement? Diabetic Medicine, 8, 706-711. doi:10.1111/j.1464-5491.1991.tb01688.x

[11] Rowe, D.J., Dawnay, A. and Watts, G.F. (1990) Microalbuminuria in diabetic subjects. Annals of Clinical Biochemistry, 27, 297-312.

[12] Amos, A., McCarty, D. and Zimmet, P. (1997) The rising global burden of diabetes and its complications: Estimates and projections to the year 2010. Diabetic Medicine, 14, S1-S85.

doi:10.1002/(SICI)1096-9136(199712)14:5+<S7::AID-D IA522>3.3.CO;2-I

[13] Zimmet, P., Alberti, K.G. and Shaw, J. (2001) Global and societal implications of the diabetes epidemic. Nature, 414, 782-787. doi:10.1038/414782a

[14] Ritz, E., Rychlik, I., Locatelli, F. and Halimi, S. (1999) End-stage renal failure in type 2 diabetes: A medical catastrophe of worldwide dimensions. American Journal of Kidney Diseases, 34, 795-808. doi:10.1016/S0272-6386(99)70035-1

[15] Ritz, E. and Orth, S.R. (1999) Nephropathy in patients with type 2 diabetes mellitus. The New England Journal of Medicine, 341, 1127-1133. doi:10.1056/NEJM199910073411506

[16] Parving, H.H. (2001) Diabetic nephropathy: Prevention and treatment. Kidney International, 60, 2041-2055. doi:10.1046/j.1523-1755.2001.00020.x

[17] Verhave, J.C., Gansevoort, R.T., Hillege, H.L., Bakker, S.J., De Zeeuw, D., de Jong, P.E., PREVEND Study
Group (2004) An elevated urinary albumin excretion predicts de novo development of renal function impairment in the general population. Kidney International, 66, S18-S21. doi:10.1111/j.1523-1755.2004.09205.X

[18] Atkins, R.C., Briganti, E.M. and Lewis, J.B. (2005) Proteinuria reduction and progression to renal failure in patients with type 2 diabetes mellitus and overt nephropathy. American Journal of Kidney Diseases, 45, 281-287. doi:10.1053/j.ajkd.2004.10.019

[19] Bruno, G., Cavallo-Perin, P., Bargero, G., Borra, M., Calvi, V. and D'Errico, N. (1996) Prevalence and risk factors for micro- and macroalbuminuria in an Italian populationbased cohort of NIDDM subjects. Diabetes Care, 19, 4347. doi:10.2337/diacare.19.1.43

[20] Parving, H.H., Lewis, J.B., Ravid, M., Remuzzi, G. and Hunsicker, L.G. (2006) DEMAND investigators. Prevalence and risk factors for microalbuminuria in a referred cohort of type II diabetic patients: A global perspective. Kidney International, 69, 2057. doi:10.1038/sj.ki.5000377

[21] Toti, F., Bejtja, G., Hoti, K., Shota, E. and Agaçi, F. (2007) Poor control and management of cardiovascular risk factors among Albanian diabetic adult patients. Primary Care Diabetes, 1, 81-86. doi:10.1016/j.pcd.2007.04.009

[22] Mather, H.M., Chaturvedi, N. and Kehely, A.M. (1998) Comparison of prevalence and risk factors for microalbuminuria in south Asians and European with type 2 diabetes mellitus. Diabetic Medicine, 15, 672-677. doi:10.1002/(SICI)1096-9136(199808)15:8<672::AID-DI A648>3.0.CO;2-3

[23] Retnakaran, R., Cull, C.A., Thorne, K.L., Adler, A.L. and Holman, R.R. (2006) Risk factors for renal dysfunction in type 2 diabetes: UK prospective diabetes study 74. Diabetes, 55, 1832-1839. doi:10.2337/db05-1620

[24] Nilsson, P.M., Gudbjornsdottir, S., Eliasson, B., Cederholm, J., Steerng Committee of the Swedish National Diabetes Register (2004) Smoking is associated with increased HbA1c values and microalbuminuria in patients with diabetes data from the National Diabetes Register in Sweden. Diabetes \& Metabolism, 30, 261-268. doi:10.1016/S1262-3636(07)70117-9

[25] Gall, M.A., Rossing, P., Scott, P., Damsbo, P., Vaag, A. and Bech, K. (1991) Prevalence of micro- and macroalbuminuria, arterial hypertension, retinopathy and large vessel disease in European type 2 (non-insulin-dependent) diabetic patients. Diabetologia, 34, 655-661. doi:10.1007/BF00400995

[26] Caramori, M.L., Fioretto, P. and Mauer, M. (2000) The need for early predictors of diabetic nephropathy risk: Is albumin excretion rate sufficient? Diabetes, 49, 13991408. doi:10.2337/diabetes.49.9.1399 